following acute viral infections. Brain Dev September 2004;26:377-379). (Respond: Dr H Yamamoto by E-mail: h3yama@marriana-u.ac.jp).

COMMENT. The authors cite 2 previous references to the remission of infantile spasms in West syndrome following viral infection, especially exanthem subitum (West, 1841; Hattori, 2001). Exanthem subitum and infection with human herpesvirus (HHV)-6 accounts for one-third of all first-time febrile seizures in children $<2$ years old in the US (Hall, Epstein, et al.1994). The virus that causes seizures may also have a protective effect in some patients. An elevated cytokine response independent of the severity of infection may be a factor in the mechanism of seizures (Kawada et al, 2003; Millichap JG, Millichap JJ, 2003), and suppression of immunological processes by anti-inflammatory cytokines (eg interleukin-10) may explain the seizure remission following viral infection in the above casereports. Further studies of the role of viral infections in the cause and remission of seizures are needed. (See Ped Neur Briefs April 2001, and Sept 2003, for further comment on cytokines and seizures).

\title{
CARDIAC IRREGULARITIES AND TEMPORAL LOBE EPILEPSY
}

Ictal and peri-ictal heart rate (HR) abnormalities in 20 children and adolescents with medically refractory symptomatic temporal lobe epilepsy (TLE) were investigated at the University Hospital Vienna, Austria. None had a known cardiac abnormality, and all were at rest in bed during EKG and EEG long-term monitoring. Of 72 TLSs analyzed, ictal tachycardia occurred in $71(98 \%)$, and ictal bradycardia was not observed. In pre-ictal stages, tachycardia occurred in 20 seizures and mild bradycardia in 3 . Tachycardia persisted $>60$ seconds after EEG seizure termination in 44 seizures (62\%). Rhythm and conduction abnormalities, including bradycardia, extreme HR oscillation, and premature ventricular beats, occurred in the postictal period during some complex partial TLSs. Early and high HR increase was associated with right mesial TLSs. The results confirm a right hemispheric lateralization of sympathetic cardiac regulation. (Mayer H, Benninger F, Urak L, et al. EKG abnormalities in children and adolescents with symptomatic temporal lobe epilepsy. Neurology July (2 of 2) 2004;63:324-328). (Reprints: Dr M Feucht, Universitatsklinik fur Neuropsychiatrie des Kindes- and Jugendalters, Wahringer Gurtel 18-20, 1090 Vienna, Austria).

COMMENT. Ictal tachycardia is the most frequent cardiac irregularity associated with complex partial seizures, occurring especially with epileptiform discharges lateralized to the right temporal lobe. It is suggested that EKG abnormalities and cardiac dysregulation during symptomatic temporal lobe epilepsy may contribute to the mechanism of sudden unexplained death.

The effect of hippocampal sclerosis on cardiovascular autonomic regulation was studied in 8 TLE patients with and 31 without hippocampal sclerosis (HS), using cardiovascular reflex tests and spectral analysis of 24-h ECG recordings, at University of Oulu, Finland. Compared to controls, a diminished heart rate variability (HRV) was observed in patients with, or without HS. Functional rather than structural changes are important in the altered cardiovascular regulation in patients with TLE. (Ansakorpi $\mathrm{H}$ et al. Epilepsia Aug 2004;45:933-939). 